\title{
Editorial comment: understanding the cost of bladder preservation in muscle invasive bladder cancer - an evaluation of radical cystectomy versus trimodal therapy costs
}

\author{
Diana E. Magee, Girish S. Kulkarni \\ Division of Urology, Department of Surgery, University Health Network, University of Toronto, ON, Canada \\ Correspondence to: Dr. Girish S. Kulkarni, MD, PhD. Division of Urology, University Health Network, 700 University Avenue, Suite 6-824, Toronto, \\ ON M5G 2M9, Canada. Email: Girish.Kulkarni@uhn.ca. \\ Provenance: This is an invited article commissioned by the Section Editor Xiao Li (Department of Urology, Jiangsu Cancer Hospital \& Jiangsu \\ Institute of Cancer Research \& Nanjing Medical University Affiliated Cancer Hospital, Nanjing, China). \\ Comment on: Williams SB, Shan Y, Ray-Zack MD, et al. Comparison of Costs of Radical Cystectomy vs Trimodal Therapy for Patients With \\ Localized Muscle-Invasive Bladder Cancer. JAMA Surg 2019:e191629. [Epub ahead of print].
}

Submitted Sep 16, 2019. Accepted for publication Oct 06, 2019.

doi: $10.21037 /$ tau.2019.10.09

View this article at: http://dx.doi.org/10.21037/tau.2019.10.09

Bladder cancer represents a significant source of morbidity and mortality worldwide. Nearly 430,000 diagnoses of bladder cancer are made each year leading to approximately 165,000 deaths (1). Within the context of healthcare spending it is a costly diagnosis and has been reported as the most expensive diagnosis per patient lifetime among all cancers (2,3), with a total cost of almost $\$ 4$ billion annually in the United States in 2010 (4). Previous studies have shown that radical cystectomy (RC) accounts for the largest proportion of costs associated with bladder cancer care (5); however, few studies have evaluated the cost of trimodal therapy (TMT). TMT has progressively been accepted as a viable treatment option for the treatment of muscle-invasive bladder cancer (MIBC) (6-8) and therefore the implications from a healthcare economic perspective have become increasingly important to consider.

In their article entitled "Comparison of Costs of Radical Cystectomy vs Trimodal Therapy for Patients With Localized Muscle-Invasive Bladder Cancer", published in FAMA Surgery, Williams and colleagues evaluated the 1 -year costs associated with the two treatment modalities (9). This retrospective cohort study utilized the Surveillance, Epidemiology, and End Results (SEER)-linked Medicare database to evaluate the overall cost of treatment for patients diagnosed with T2-T4aN0M0 urothelial bladder cancer in older adults (age 66-85). Patients were followed from the time of diagnosis, defined as the transurethral resection of the bladder tumour (TURBT) immediately preceding RC or TMT, for 1 year or until death. TMT was defined by the receipt of a TURBT followed by radiation treatment and chemotherapy within 180 days following diagnosis in the absence of a RC.

Overall, 2,963 patients with MIBC were included in the analysis; of those, $2,235(75.4 \%)$ and $728(24.6 \%)$ were treated with RC and TMT, respectively. In the raw analysis, those treated with $\mathrm{RC}$ were younger, more likely to be female, less comorbid and have more advanced tumour stages. After propensity adjustments using inverse probability treatment weights all variables were well balanced. They found higher 1-year median costs for patients undergoing TMT (\$289,142, IQR: \$197,649$409,655)$ compared to RC $(\$ 148,757$, IQR: $\$ 87,282-$ 252,518). Costs for the initial 3-month period between diagnosis and treatment were roughly the same for the two treatment cohorts, however the cost disparity continued to widen over the course of the study year. At 90 days, total TMT costs were $\$ 83,754$ (IQR: $\$ 50,754-129,299$ ) versus $\$ 68,692$ (IQR: $\$ 44,912-98,871$ ) for RC whereas at 180 days they were $\$ 187,162$ (IQR: $\$ 126,905-261,817$ ) versus \$109,078 (IQR: \$71,368-170,788), respectively. Moreover, they found that overall survival (HR: 1.54, 95\% CI: 1.39-1.71) was lower for patients receiving TMT; however, this was attenuated when accounting for number of fractions of radiation received ( $\geq 27$ fractions: OS HR: 
1.22, 95\% CI: 1.09-1.37 vs. <27 fractions: OS HR: 1.59 , $95 \%$ CI: 1.41-1.79). The authors conclude that the use of TMT rather than RC would result in an excess spending of approximately $\$ 135,000$ per patient per year in the context of lower overall and cancer-specific survival.

The authors should be congratulated for this important work in an understudied aspect of bladder cancer care using a large and well-established administrative dataset. Their study, the largest thus far evaluating the costs of TMT, further illustrates the significant impact that MIBC care has on the healthcare economic landscape. The cost analysis from this study is largely consistent with results from their earlier work which evaluated the costs at 30,90 and 180 days post RC and TMT in 687 propensity matched patients from the same SEER cohort (10).

The authors' results and interpretations should however, be approached with caution considering this study's limitations. The use of administrative data, while providing a large sample size, does not easily lend itself to answering questions regarding TMT (11). The costs for TMT at each interval are remarkably higher than those in the $\mathrm{RC}$ arm. In the period between 90 and 180 days and between 180 and 365 days the cost to treat patients in the TMT arm was over $\$ 100,000$ per period, compared to approximately $\$ 40,000$ in the RC group over the same phases. While it is accepted that patients treated with TMT will require ongoing cystoscopic surveillance $(6,7)$ along with routine imaging, the discrepancy in cost between these two groups is difficult to justify from only routine urologic care especially considering only $3.4 \%$ of the TMT group required a salvage cystectomy. Although the authors note that the extra costs for the TMT group are related to medication and radiology expenses during follow up, it leaves us to wonder what additional radiologic and medication interventions occurred in TMT patients to sum to $\$ 135,000$ in excess? Unfortunately, these granular data were unavailable to report.

The elevated costs for TMT follow-up may, on the other hand, be explained by the methodology by which they calculated the costs. Their study used overall Medicare expenditures (rather than cancer-specific costs) which has the potential to introduce unmeasured confounding. If patients in the TMT arm were less likely to receive a RC due to underlying comorbidity status, then they were also more likely to incur costs for treatment of their comorbidities as time progresses which would have resulted in increased overall Medicare spending. The propensity score adjustments are helpful in accounting for this but do not entirely address the potential underlying confounders. Differences in comorbidity status also have implications with respect to the OS and cancer-specific survival results, as patients who receive TMT as an option of last resort would certainly bias the results towards poorer outcomes and higher costs (11).

Moreover, the significant difference in ongoing care costs for patients treated with RC versus TMT may be explained by the limitations around the identification of TMT patients. First, ideal candidates for TMT should meet strict criteria including small, solitary muscleinvasive tumours with no significant carcinoma-in-situ, and who have undergone a complete TURBT without visible evidence of residual disease (12). However, using the SEER database it is difficult to determine if the patients who received TMT met these exacting inclusion criteria. Complete resection of the tumour is a strong predictor of TMT success, but it is impossible to determine quality of resection from population-level data (13). Second, the quality of the chemotherapy regimens is unknown. Ideally, chemotherapy for TMT should be cisplatin-based and administered concomitantly with radiation therapy (12). However, the type and timing of chemotherapy is not discussed and therefore it is difficult to determine whether the TMT was in fact, complete. If complete TURBTs were not done or appropriate chemotherapy regimens were not received or completed this would certainly bias the results towards poorer outcomes and higher costs due to treatment failures. It also remains entirely possible that some of the TMT patients received sequential radiation followed by chemotherapy with palliative rather than curative intent based on tumour and patient characteristics. This is especially true considering in the era studied, TMT was rarely used as primary therapy and not well accepted so the patients receiving TMT may not represent those who received TMT with curative intent. Given that the costs of palliation and death from cancer are extraordinarily high (14), this possibility would introduce significant cost bias against the TMT group.

The authors suggest that improved survival and a gain of quality-adjusted life years are necessary to justify the use of TMT. While we agree with the authors that incorporating quality-of-life assessments into the evaluation of RC versus TMT is a worthwhile pursuit, TMT as a treatment option should not be abandoned based on the results presented by Williams et al. Phase specific cost assessments, rather than purely time based assessments, should be considered as they better incorporate the natural history of the disease 
and the corresponding patterns of treatment leading to more granular cost information (14). Furthermore, cancerspecific costs should be collected rather than relying on allcause billings. While conflicting evidence exists regarding the efficacy of TMT compared to RC $(8,15)$, the current cost data should not dissuade practitioners. Therefore, we continue to advocate that bladder preservation is a worthy pursuit in the treatment of MIBC in well-selected and informed patients.

\section{Acknowledgments}

None.

\section{Footnote}

Conflicts of Interest: The authors have no conflicts of interest to declare.

Etbical Statement: The authors are accountable for all aspects of the work in ensuring that questions related to the accuracy or integrity of any part of the work are appropriately investigated and resolved.

\section{References}

1. Ferlay J, Soerjomataram I, Dikshit R, et al. Cancer incidence and mortality worldwide: sources, methods and major patterns in GLOBOCAN 2012. Int J Cancer 2015;136:E359-86.

2. Botteman MF, Pashos CL, Redaelli A, et al. The health economics of bladder cancer: a comprehensive review of the published literature. Pharmacoeconomics 2003;21:1315-30.

3. Yeung C, Dinh T, Lee J. The health economics of bladder cancer: an updated review of the published literature. Pharmacoeconomics 2014;32:1093-104.

4. Cooksley CD, Avritscher EB, Grossman HB, et al. Clinical model of cost of bladder cancer in the elderly. Urology

\footnotetext{
Cite this article as: Magee DE, Kulkarni GS. Editorial comment: understanding the cost of bladder preservation in muscle invasive bladder cancer-an evaluation of radical cystectomy versus trimodal therapy costs. Transl Androl Urol 2019;8(Suppl 5):S463-S465. doi: 10.21037/tau.2019.10.09
}

2008;71:519-25.

5. Mossanen M, Gore JL. The burden of bladder cancer care: direct and indirect costs. Curr Opin Urol 2014;24:487-91.

6. Kulkarni GS, Black PC, Sridhar SS, et al. Canadian Urological Association guideline: Muscle-invasive bladder cancer. Can Urol Assoc J 2019:230-8.

7. Alfred Witjes J, Lebret T, Compérat EM, et al. Updated 2016 EAU Guidelines on Muscle-invasive and Metastatic Bladder Cancer. European Urology 2017;71:462-75.

8. Kulkarni GS, Hermanns T, Wei Y, et al. Propensity Score Analysis of Radical Cystectomy Versus Bladder-Sparing Trimodal Therapy in the Setting of a Multidisciplinary Bladder Cancer Clinic. J Clin Oncol 2017;35:2299-305.

9. Williams SB, Shan Y, Ray-Zack MD, et al. Comparison of Costs of Radical Cystectomy vs Trimodal Therapy for Patients With Localized Muscle-Invasive Bladder Cancer. JAMA Surg 2019:e191629.

10. Williams SB, Shan Y, Jazzar U, et al. Comparing Survival Outcomes and Costs Associated With Radical Cystectomy and Trimodal Therapy for Older Adults With MuscleInvasive Bladder Cancer. JAMA Surg 2018;153:881-9.

11. Kulkarni GS, Klaassen Z. Trimodal Therapy is Inferior to Radical Cystectomy for Muscle-invasive Bladder Cancer using Population-level Data: Is There Evidence in the (Lack of) Details? Eur Urol 2017;72:488-9.

12. Chen RC, Shipley WU, Efstathiou JA, et al. Trimodality bladder preservation therapy for muscle-invasive bladder cancer. J Natl Compr Canc Netw 2013;11:952-60.

13. Efstathiou JA, Spiegel DY, Shipley WU, et al. Long-term outcomes of selective bladder preservation by combinedmodality therapy for invasive bladder cancer: the MGH experience. Eur Urol 2012;61:705-11.

14. de Oliveira C, Pataky R, Bremner KE, et al. Phase-specific and lifetime costs of cancer care in Ontario, Canada. BMC Cancer 2016;16:809.

15. Seisen T, Sun M, Lipsitz SR, et al. Comparative Effectiveness of Trimodal Therapy Versus Radical Cystectomy for Localized Muscle-invasive Urothelial Carcinoma of the Bladder. Eur Urol 2017;72:483-7. 